\title{
On Universal Point Sets for Planar Graphs
}

\author{
Jean Cardinal ${ }^{1, *} \quad$ Michael Hoffmann ${ }^{2, \dagger} \quad$ Vincent Kusters ${ }^{2, \dagger}$ \\ ${ }^{1}$ Département d'Informatique, Université Libre de Bruxelles, jcardin@ulb.ac.be \\ ${ }^{2}$ Institute of Theoretical Computer Science, ETH Zürich, \{hoffmann, kustersv\}@inf.ethz.ch
}

\begin{abstract}
A set $P$ of points in $\mathbb{R}^{2}$ is $n$-universal, if every planar graph on $n$ vertices admits a plane straight-line embedding on P. Answering a question by Kobourov, we show that there is no $n$-universal point set of size $n$, for any $n \geq 15$. Conversely, we use a computer program to show that there exist universal point sets for all $n \leq 10$ and to enumerate all corresponding order types. Finally, we describe a collection $\mathcal{G}$ of $7^{\prime} 393$ planar graphs on 35 vertices that do not admit a simultaneous geometric embedding without mapping, that is, no set of 35 points in the plane supports a plane straight-line embedding of all graphs in $\mathcal{G}$.
\end{abstract}

\section{Introduction}

We consider plane, straight-line embeddings of graphs in $\mathbb{R}^{2}$. In those embeddings, vertices are represented by pairwise distinct points, every edge is represented by a line segment connecting its endpoints, and no two edges intersect except at a common endpoint.

An $n$-universal (or short universal) point set for planar graphs admits a plane straight-line embedding of all graphs on $n$ vertices. A longstanding open problem is to give precise bounds on the minimum number of points in an n-universal point set. The currently known asymptotic bounds are apart by a linear factor. On the one hand, it is known that every planar graph can be embedded on a grid of size $n-1 \times n-1$ [9, 17]. On the other hand, it was shown that at least $1.235 n$ points are necessary [15], improving earlier bounds of $1.206 n[8]$ and $n+\sqrt{n}[9]$.

The following, somewhat simpler question was asked ten years ago by Kobourov [10]: what is the largest value of $n$ for which a universal point set of size $n$ exists? We prove the following.

Theorem 1 There is no $n$-universal point set of size $n$, for any $n \geq 15$.

At some point, the Open Problem Project page dedicated to the problem [10] mentioned that Kobourov proved there exist 14-universal point sets of size 14. If this is correct, our bound is tight, and the answer to the above question is $n=14$. After verification, however, this claim appears to be unsubstantiated [14]. We managed to check that there exist universal point sets only up to $n \leq 10$. Further investigations are ongoing.

\footnotetext{
${ }^{*}$ Partially supported by the ESF EUROCORES programme EuroGIGA, CRP ComPoSe. The research was carried out while the first author was at ETH Zürich.

${ }^{\dagger}$ Partially supported by the ESF EUROCORES programme EuroGIGA, CRP GraDR and the Swiss National Science Foundation, SNF Project 20GG21-134306.
} 
Overview. Section 2 is devoted to the proof of Theorem 1. It combines a labeled counting scheme for planar 3-trees (also known as stacked triangulations) that is very similar to the one used by Kurowski in his asymptotic lower bound argument [15] with known lower bounds on the rectilinear crossing number [1, 16]. Note that although planar 3-trees seem to be useful for lower bounds, a recent preprint from Fulek and Tóth [12] shows that there exist $n$-universal point sets of size $\mathrm{O}\left(\mathrm{n}^{5 / 3}\right)$ for planar 3-trees.

For a collection $\mathcal{G}=\left\{\mathrm{G}_{1}, \ldots, \mathrm{G}_{\mathrm{k}}\right\}$ of planar graphs on $\mathrm{n}$ vertices, a simultaneous geometric embedding without mapping for $\mathcal{G}$ is a collection of plane straight-line embeddings $\phi_{i}: G_{i} \rightarrow P$ onto the same set $\mathrm{P} \subset \mathbb{R}^{2}$ of $n$ points.

In Section 3, we consider the following problem: what is the largest natural number $\sigma$ such that every collection of $\sigma$ planar graphs on the same number of vertices admit a simultaneous geometric embedding without mapping? From the Fáry-Wagner Theorem [11, 18] we know that $\sigma \geq 1$. We prove the following upper bound:

Theorem 2 There is a collection of 7'393 planar graphs on 35 vertices that do not admit a simultaneous plane straight-line embedding without mapping, hence $\sigma<7^{\prime} 393$.

To our knowledge these are the best bounds currently known. It is a very interesting and probably challenging open problem to determine the exact value of $\sigma$.

Finally, in Section 4, we use a computer program to show that there exist $n$-universal point sets of size $n$ for all $n \leq 10$ and give the total number of such point sets for each $n$. As a side remark, note that it is not clear that the property "there exists an n-universal point set of size $n$ " is monotone in $n$.

\section{Large Universal Point Sets}

A planar 3-tree is a maximal planar graph obtained by iteratively splitting a facial triangle into three new triangles with a degree-three vertex, starting from a single triangle. Since a planar 3-tree is a maximal planar graph, it has $n$ vertices and $2 n-4$ triangular faces and its combinatorial embedding is fixed up to the choice of the outer face.

For every integer $n \geq 4$, we define a family $\mathcal{T}_{n}$ of labeled planar 3-trees on the set of vertices $[\mathrm{n}]:=\{1, \ldots, \mathrm{n}\}$ as follows:

(i) $\mathcal{T}_{4}$ contains only the complete graph $\mathrm{K}_{4}$,

(ii) $\mathcal{T}_{n}$ contains every graph that can be constructed by making the new vertex $n$ adjacent to the three vertices of one of the $2 n-6$ facial triangles of some $T \in \mathcal{T}_{n-1}$.

We insist on the fact that $\mathcal{T}_{\mathrm{n}}$ is a set of labeled abstract graphs, many of which can in fact be isomorphic if considered as abstract (unlabeled) graphs. We also point out that for $n>4$, the class $\mathcal{T}_{\mathfrak{n}}$ does not contain all labeled planar 3 -trees on $n$ vertices. For instance, the four graphs in $\mathcal{T}_{5}$ are shown in Figure 1, and there is no graph for which both Vertex 1 and Vertex 2 have degree three.

Lemma 3 For $n \geq 4$, we have $\left|\mathcal{T}_{n}\right|=2^{n-4} \cdot(n-3)$ !.

Proof. By definition, $\left|\mathcal{T}_{4}\right|=1$. Every graph in $\mathcal{T}_{n}$ is constructed by splitting one of the $2 n-6$ faces of a graph in $\mathcal{T}_{\mathrm{n}-1}$. We therefore have:

$$
\left|\mathcal{T}_{n}\right|=\left|\mathcal{T}_{n-1}\right| \cdot(2 n-6)=4 \cdot 6 \cdot \ldots \cdot(2 n-6)=2^{n-4} \cdot(n-3) !
$$



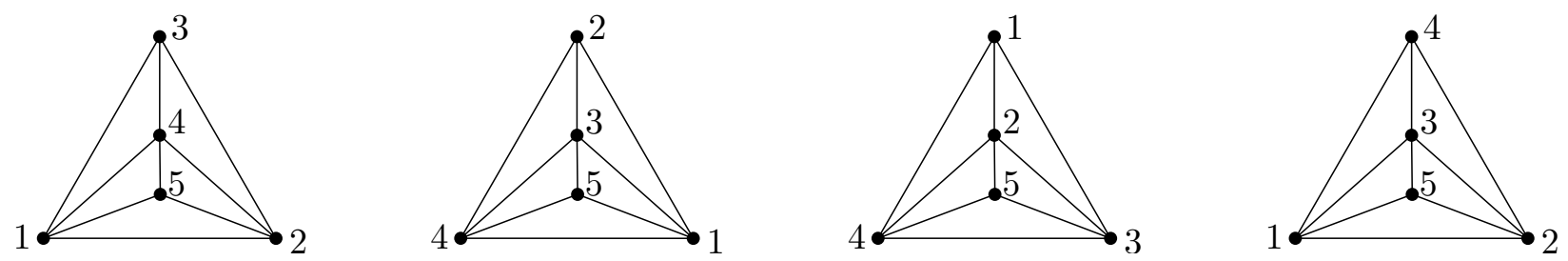

Figure 1: The four planar 3 -trees in $\mathcal{T}_{5}$, with vertex set $\{1,2,3,4,5\}$.

Lemma 4 Given a set $\mathrm{P}=\left\{\mathrm{p}_{1}, \ldots, \mathrm{p}_{\mathrm{n}}\right\}$ of labeled points in the plane and a bijection $\pi:[\mathrm{n}] \rightarrow \mathrm{P}$, there is at most one $\mathrm{T} \in \mathcal{T}_{\mathrm{n}}$ such that $\pi$ is a plane straight-line embedding of $\mathrm{T}$.

Proof. Consider any such labeled point set $P$ and assume without loss of generality that $\pi(i)=p_{i}$ for all $i$. In all $T \in \mathcal{T}_{\mathrm{n}}$ the vertices $\{1,2,3,4\}$ form a $K_{4}$. Hence, for all $T$, the straight-line embedding $\pi$ connects all pairs of points in $\left\{p_{1}, p_{2}, p_{3}, p_{4}\right\}$ with line segments. If these points are in convex position, there is a crossing and there is no $T \in \mathcal{T}_{\mathrm{n}}$ for which $\pi$ is a plane straight-line embedding (Figure 2). Otherwise, there is a unique graph $\mathrm{K}_{4} \in \mathcal{T}_{4}$ for which $\mathrm{p}_{1}, \mathrm{p}_{2}, \mathrm{p}_{3}, \mathrm{p}_{4}$ is a plane straight-line drawing. We proceed as follows.
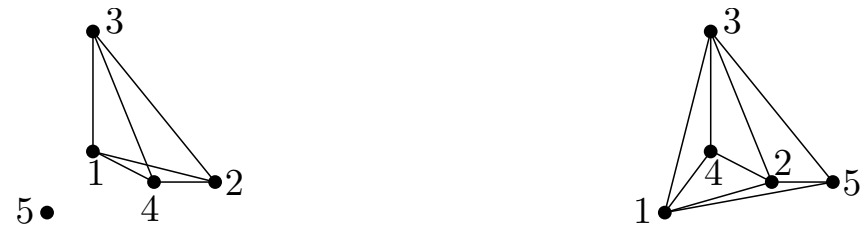

Figure 2: Some permutations of a given point set do not define any planar 3-tree in $\mathcal{T}_{n}$, because they generate a crossing (left). On the other hand, when no such crossing occurs, the permutation defines a unique planar 3 -tree in $\mathcal{T}_{\mathrm{n}}$ (right). At any rate, a single permutation can be associated with at most one planar 3 -tree in $\mathcal{T}_{n}$.

Given a plane straight-line drawing for some graph $T_{i} \in \mathcal{T}_{i}$ on the first $i \geq 4$ points, the next point $p_{i+1}$ is located in some triangular region of the drawing; denote this region by $p_{a_{i}} p_{b_{i}} p_{c_{i}}$. Only if during the construction of $T$ we decided to connect the next vertex $i+1$ to exactly the vertices $a_{i}, b_{i}, c_{i}$, there is no crossing introduced by mapping $i+1$ to $p_{i+1}$. (An edge to any other vertex would cross one of the bounding edges of the triangle $p_{a_{i}} p_{b_{i}} p_{c_{i}}$.) In other words, for every $i \geq 5$ the role of vertex $i$ is completely determined. If no crossing is ever introduced, this process determines exactly one graph $T \in \mathcal{T}_{n}$ for which $\pi$ forms a plane straight-line embedding. (Note that a crossing can be introduced only if $p_{i+1}$ is located outside of the convex hull of $\left\{p_{1}, \ldots, p_{i}\right\}$. And also in that case there need not be a crossing, as the example in Figure 2 (right) shows.)

We use the following theorem by Ábrego and Fernández-Merchant.

Theorem $5([1])$ Every plane straight-line drawing of the complete graph $\mathrm{K}_{\mathrm{n}}$ has at least $\frac{1}{4}\left\lfloor\frac{n}{2}\right\rfloor\left\lfloor\frac{n-1}{2}\right\rfloor\left\lfloor\frac{n-2}{2}\right\rfloor\left\lfloor\frac{n-3}{2}\right\rfloor$ crossings.

Note that for $n \leq 4$ at least one of the floor expressions is zero, whereas for $n=5$ the theorem states that every straight-line drawing of $\mathrm{K}_{5}$ has at least one crossing. Any pair of crossing edges corresponds to a four-tuple of points in convex position. Using this interpretation we can easily 
derive a floor-free lower bound on the number of convex four-gons contained in every planar point set.

Corollary 6 Given a point set $\mathrm{P} \subset \mathbb{R}^{2}$ of $\mathrm{n}$ points in general position, more than $a \frac{3}{8} \cdot \frac{\mathrm{n}-4}{\mathrm{n}}$-fraction of all four element subsets of $\mathrm{P}$ is in convex position.

Proof. By Theorem 5 at least $c=\frac{1}{4}\left\lfloor\frac{n}{2}\right\rfloor\left\lfloor\frac{n-1}{2}\right\rfloor\left\lfloor\frac{n-2}{2}\right\rfloor\left\lfloor\frac{n-3}{2}\right\rfloor$ four element subsets of $P$ are in convex position. For $n$ odd we have

$$
c=\frac{1}{4}\left(\frac{n-1}{2}\right)^{2}\left(\frac{n-3}{2}\right)^{2}
$$

and for $\mathrm{n}$ even we have

$$
c=\frac{1}{4}\left(\frac{n}{2}\right)\left(\frac{n-2}{2}\right)^{2}\left(\frac{n-4}{2}\right)
$$

and so

$$
\begin{aligned}
c & >\frac{1}{4}\left(\frac{n-1}{2}\right)\left(\frac{n-2}{2}\right)\left(\frac{n-3}{2}\right)\left(\frac{n-4}{2}\right) \\
& =\frac{3}{8} \cdot \frac{n-4}{n} \cdot \frac{n(n-1)(n-2)(n-3)}{4 \cdot 3 \cdot 2} \\
& =\frac{3}{8} \cdot \frac{n-4}{n} \cdot\left(\begin{array}{l}
n \\
4
\end{array}\right),
\end{aligned}
$$

for all $n$.

We will use this fact to prove the following lemma.

Lemma 7 On any set $\mathrm{P} \subset \mathbb{R}^{2}$ of $\mathrm{n} \geq 4$ points fewer than $\frac{1}{8}(5 n+12)(\mathrm{n}-1)$ ! graphs from $\mathcal{T}_{\mathrm{n}}$ admit a plane straight-line embedding.

Proof. Let $\mathrm{P} \subset \mathbb{R}^{2}$ be a set of $\mathrm{n}$ points and denote by $\mathcal{F}_{\mathrm{n}} \subseteq \mathcal{T}_{\mathrm{n}}$ the set of labeled planar 3-trees from $\mathcal{T}_{\mathrm{n}}$ that admit a plane straight-line embedding onto $\mathrm{P}$. Note that a straight-line embedding can be represented by a permutation $\pi$ of the points of $P$, where each vertex $i$ is mapped to point $\pi(i)$. Let $S_{n}$ be the set of all permutations of P. We define a map $\psi: \mathcal{F}_{n} \rightarrow S_{n}$ by assigning to each $T \in \mathcal{F}_{n}$ some $\psi(T) \in S_{n}$ such that $\psi(T)$ is a plane straight-line embedding of $T$ (such an embedding exists by definition of $\mathcal{F}_{n}$ ).

By Lemma 4, every permutation $\pi \in S_{n}$ is a plane straight-line embedding of at most one $\mathrm{T} \in \mathcal{F}_{\mathrm{n}}$. It follows that $\psi$ is a injection, and hence $\psi: \mathcal{F}_{\mathrm{n}} \rightarrow \Pi$, with $\Pi=\operatorname{Im}(\psi)$, is a bijection and so $\left|\mathcal{F}_{n}\right|=|\Pi| \leq\left|S_{n}\right|=n !$.

Next we can quantify the difference between $\Pi$ and $S_{\mathfrak{n}}$ using Corollary 6 . Note that the general position assumption is not a restriction, since in case of collinearities, a slight perturbation of the point set yields a new point set that still admits all plane straight-line drawings of the original point set. Consider a permutation $\pi=p_{1}, \ldots, p_{n}$ such that $p_{1}, p_{2}, p_{3}, p_{4}$ form a convex quadrilateral. As argued in the first paragraph of the proof of Lemma 4 $\pi$ is not a plane straight-line embedding for any $T \in \mathcal{F}_{n}$. It follows that $\pi \in S_{n} \backslash \Pi$. We know from Corollary 6 that more than a fraction of $(3 / 8) \cdot(n-4) / n$ of the 4 -tuples of $\mathrm{P}$ are in convex position and therefore a corresponding fraction 
of all permutations does not correspond to a plane straight-line drawing. So we can bound the number of possible labeled plane straight-line drawings by

$$
|\Pi|<\left(1-\frac{3}{8} \cdot \frac{n-4}{n}\right) n !=\frac{1}{8}(5 n+12)(n-1) ! .
$$

Proof. [of Theorem 1] Consider an $n$-universal point set $P \subset \mathbb{R}^{2}$ with $|P|=n$. Being universal, in particular $P$ has to accommodate all graphs from $\mathcal{T}_{n}$. By Lemma 3 , there are exactly $2^{n-4} \cdot(n-3)$ ! graphs in $\mathcal{T}_{n}$, whereas by Lemma 7 no more than $\frac{1}{8}(5 n+12)(n-1)$ ! graphs from $\mathcal{T}_{n}$ admit a plane straight-line drawing on $P$. Combining both bounds we obtain $2^{n-1} \leq(5 n+12)(n-1)(n-2)$. Setting $n=15$ yields $2^{14}=16^{\prime} 384 \leq 87 \cdot 14 \cdot 13=15^{\prime} 834$, which is a contradiction and so there is no 15 -universal set of 15 points.

For $\mathrm{n}=14$ the inequality reads $2^{13}=8^{\prime} 192 \leq 82 \cdot 13 \cdot 12=12^{\prime} 792$ and so there is no indication that there cannot be a 14-universal set of 14 points. To prove the claim for any $n>15$, consider the two functions $f(n)=2^{n-1}$ and $g(n)=(5 n+12)(n-1)(n-2)$ that constitute the inequality. As $f$ is exponential in $n$ whereas $g$ is just a cubic polynomial, $f$ certainly dominates $g$, for sufficiently large $n$. Moreover, we know that $f(15)>g(15)$. Noting that $f(n) / f(n-1)=2$ and $g(n)>0$, for $n>2$, it suffices to show that $g(n) / g(n-1)<2$, for all $n \geq 16$.

We can bound

$$
\begin{aligned}
\frac{g(n)}{g(n-1)} & =\frac{(5 n+12)(n-1)(n-2)}{(5(n-1)+12)(n-2)(n-3)}=\frac{(5 n+12)(n-1)}{(5 n+7)(n-3)} \\
& <\frac{(5 n+15) n}{5 n(n-3)}=\frac{n+3}{n-3}
\end{aligned}
$$

which is easily seen to be upper bounded by two, for $n \geq 9$.

\section{Simultaneous Geometric Embeddings}

The number of non-isomorphic planar 3-trees on $n$ vertices was computed by Beineke and Pippert [3], and appears as sequence A027610 on Sloane's Encyclopedia of Integer Sequences. For $n=15$, this number is $321^{\prime} 776$. Hence we can also phrase our result in the language of simultaneous embeddings [4].

Corollary 8 There is a collection of 321'776 planar graphs that do not admit a simultaneous (plane straight-line) embedding without mapping.

In the following we will give an explicit construction for a much smaller family of graphs that not admit a simultaneous embedding without mapping. As a first observation, note that the freedom to select the outer face is essential in order to embed graphs onto a given point set. In fact, for planar 3-trees, the mapping for the outer face is the only choice there is. We prove this in two steps.

Lemma 9 Let $\mathrm{G}$ be a labeled planar 3-tree on the vertex set $[\mathrm{n}]$, for $\mathrm{n} \geq 3$, and let $\mathrm{C}$ denote any triangle in $\mathrm{G}$. Then $\mathrm{G}$ can be constructed starting from $\mathrm{C}$ by iteratively inserting $a$ degree-three vertex into some facial triangle of the partial graph constructed so far. 
Proof. We prove the statement by induction on $n$. For $n=3$ there is nothing to show. Hence let $n>3$. By definition $G$ can be constructed iteratively from some triangle in the way described. Without loss of generality suppose that adding vertices in the order $1,2, \ldots, n$ yields such a construction sequence. Denote by $G_{i}$ the graph that is constructed by the sequence $1, \ldots, i$, for $1 \leq i \leq n$.

Let $C=u, v, w$ such that $u<v<w$. Consider the graph $G_{w}$ : In the last step, $w$ is added as a new vertex into some facial triangle $T$ of $\mathrm{G}_{w-1}$. As $w$ is a neighbor of both $u$ and $v$ in $\mathrm{G}$, both $u$ and $v$ are vertices of $T$; denote the third vertex of $T$ by $x$. Note that all of $u, v, w$ and $u, w, x$ and $v, x, w$ are facial triangles in $\mathrm{G}_{w}$.

If $w=4$, then exchanging the role of $w$ and $x$ yields a construction sequence $u, v, w, x, 5, \ldots, n$ for $\mathrm{G}$, as claimed. If $w>4$, then $\mathrm{c}_{1}, \mathrm{c}_{2}, v$ is a separating triangle in $\mathrm{G}_{w}$. By the inductive hypothesis we can obtain a construction sequence $S$ for $G_{w-1}$ starting with the triangle $u, v, x$. The desired sequence for $\mathrm{G}$ is obtained as $u, v, w, x, S^{-}, w+1, \ldots, n$, where $S^{-}$is the suffix of $S$ that excludes the starting triangle $u, v, x$.

And now we can prove the desired property:

Lemma 10 Given a labeled planar 3-tree $\mathrm{G}$ on vertex set $[\mathrm{n}]$, a triangle $\mathrm{c}=\mathrm{c}_{1} \mathrm{c}_{2} \mathrm{c}_{3}$ in $\mathrm{G}$, and a set $\mathrm{P} \subset \mathbb{R}^{2}$ of $\mathrm{n}$ points with $\mathrm{p}_{1}, \mathrm{p}_{2}, \mathrm{p}_{3} \in \mathrm{P}$, there is at most one way to complete the partial embedding $\left\{\mathrm{c}_{1} \mapsto \mathrm{p}_{1}, \mathrm{c}_{2} \mapsto \mathrm{p}_{2}, \mathrm{c}_{3} \mapsto \mathrm{p}_{3}\right\}$ to a plane straight-line embedding of $\mathrm{G}$ on $\mathrm{P}$.

Proof. We use Lemma 9 to relabel the vertices in such a way that $c_{1}, c_{2}, c_{3}$ becomes $1,2,3$ and the order $1, \ldots, n$ is a construction sequence for $G$. Embed vertices 1,2,3 onto $p_{1}, p_{2}, p_{3}$. We iteratively embed the remaining vertices as follows. Vertex $i$ was inserted into some face $j k \ell$ during the construction given by Lemma 9. Note that $j, k, \ell$ have already been embedded on points $p_{j}, p_{k}, p_{\ell}$. The vertices contained in the triangle $j k \ell$ (except $i$ ) are partitioned into three sets by the cycles $i j k$ ( $n_{1}$ vertices) and $i k \ell$ ( $n_{2}$ vertices) and $i \ell j$ ( $n_{3}$ vertices). We want to embed $i$ on a point $p_{i}$ such that $p_{i} p_{j} p_{k}$ contains exactly $n_{1}$ points, $p_{i} p_{k} p_{\ell}$ contains exactly $n_{2}$ points and $p_{i} p_{\ell} p_{j}$ contains exactly $n_{3}$ points. Note that it is necessary to embed $i$ on a point with this property: if some triangle has too few points, then it will not be possible to embed the subgraph of $G$ enclosed by the corresponding cycle there. It remains to show that there is always at most one choice for $p_{i}$. Suppose that there are two candidates for $p_{i}$, say $p_{i}^{\prime}$ and $p_{i}^{\prime \prime}$. Then $p_{i}^{\prime \prime}$ must be contained in $p_{i}^{\prime} p_{j} p_{k}$ or $p_{i}^{\prime} p_{k} p_{\ell}$ or $p_{i}^{\prime} p_{\ell} p_{j}$ (or vice versa). Without loss of generality, let it be contained in $p_{i}^{\prime} p_{j} p_{k}$ : now $p_{i}^{\prime \prime} p_{j} p_{k}$ contains fewer points than $p_{i}^{\prime} p_{j} p_{k}$, which is a contradiction. The lemma follows by induction.

Therefore it is not surprising that it is very easy to find three graphs that do not admit a simultaneous (plane straight-line) embedding without mapping, if the mapping for the outer face is specified for each of them.

Lemma 11 There is no set $\mathrm{P} \subset \mathbb{R}^{2}$ of five points with convex hull $\mathrm{p}_{\mathrm{a}}, \mathrm{p}_{\mathrm{b}}, \mathrm{p}_{\mathrm{c}}$ such that every graph shown in Figure 3 has a (plane straight-line) embedding on $\mathrm{P}$ where the vertices $\mathrm{a}, \mathrm{b}$ and $\mathrm{c}$ are mapped to the points $\mathrm{p}_{\mathrm{a}}, \mathrm{p}_{\mathrm{b}}$ and $\mathrm{p}_{\mathrm{c}}$, respectively.

Proof. The point $p$ for the central vertex that is connected to all of $a, b, c$ must be chosen so that (i) it is not in convex position with $p_{a}, p_{b}$ and $p_{c}$ and (ii) the number of points in the three resulting triangles is one in one triangle and zero in the other two. That requires three distinct choices for $p$, but there are only two points available. 

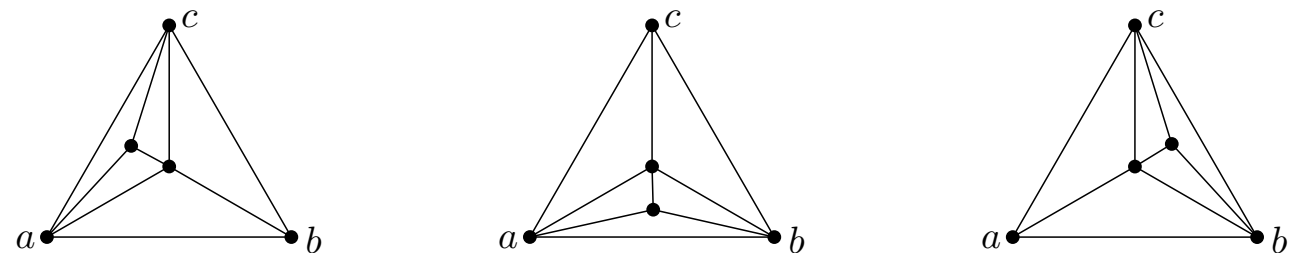

Figure 3: Three planar graphs that do not admit a simultaneous geometric embedding with a fixed mapping for the outer face.

In fact, there are many such triples of graphs. The following lemma can be verified with help of a computer program that exhaustively checks all order types. Point set order types [13] are a combinatorial abstraction of planar point sets that encode the orientation of all point triples, which in particular determines whether or not any two line segments cross. For a small number of points, there is a database with realizations of every (realizable) order type [2].

Lemma 12 There is no set $\mathrm{P} \subset \mathbb{R}^{2}$ of eight points with convex hull $\mathrm{p}_{\mathrm{a}}, \mathrm{p}_{\mathrm{b}}, \mathrm{p}_{\mathrm{c}}$ such that every graph shown in Figure 4 has a (plane straight-line) embedding on $\mathrm{P}$ where the vertices $\mathrm{a}, \mathrm{b}$ and $\mathrm{c}$ are mapped to the points $\mathrm{p}_{\mathrm{a}}, \mathrm{p}_{\mathrm{b}}$ and $\mathrm{p}_{\mathrm{c}}$, respectively.

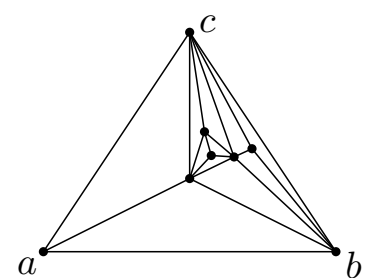

(a) $T_{1}$

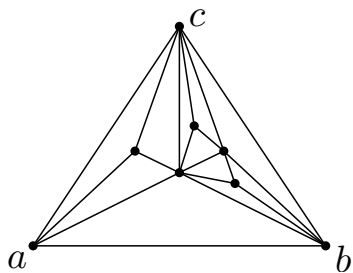

(e) $T_{5}$

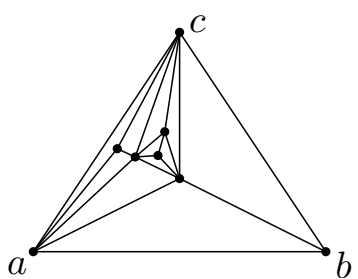

(b) $T_{2}$

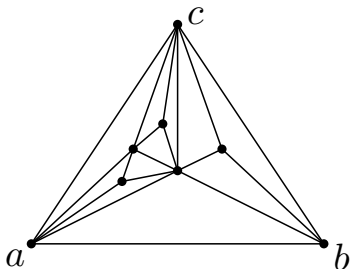

(f) $T_{6}$

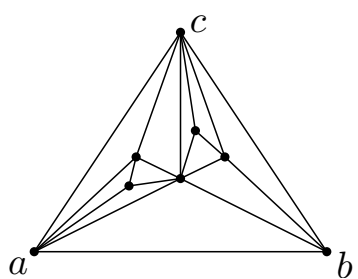

(c) $T_{3}$

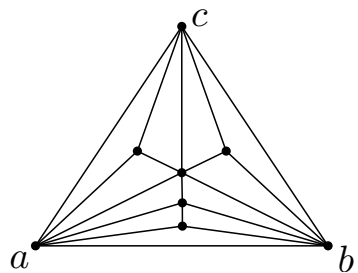

(g) $T_{7}$

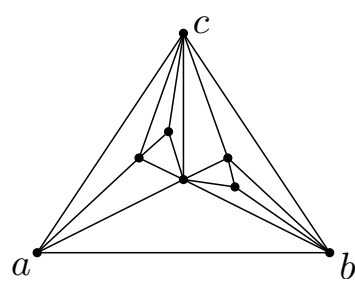

(d) $T_{4}$

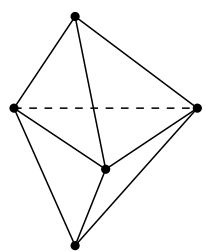

(h) B

Figure 4: (a)-(g): Seven planar graphs, no three of which admit a simultaneous geometric embedding with a fixed mapping for the outer face; $(\mathrm{h})$ : the skeleton $\mathrm{B}$ of a triangular bipyramid.

Denote by $\mathcal{T}=\left\{T_{1}, \ldots, T_{7}\right\}$ the family of seven graphs on eight vertices depicted in Figure 4 . We consider these graphs as abstract but rooted graphs, that is, one face is designated as the outer face and the counterclockwise order of the vertices along the outer face (the orientation of the face) is $a, b, c$ in each case. Observe that all graphs in $\mathcal{T}$ are planar 3-trees.

Using $\mathcal{T}$ we construct a family $\mathcal{G}$ of graphs as follows. Start from the skeleton B of a triangular bipyramid, that is, a triangle and two additional vertices, each of which is connected to all vertices of the triangle. The graph B has five vertices and six faces and it is a planar 3-tree. 
We obtain $\mathcal{G}$ from $\mathrm{B}$ by planting one of the graphs from $\mathcal{T}$ onto each of the six faces of $\mathrm{B}$. Each face of $B$ is a (combinatorial) triangle where one vertex has degree three (one of the pyramid tips) and the other two vertices have degree four (the vertices of the starting triangle). On each face $f$ of $B$ a selected graph $T$ from $\mathcal{T}$ is planted by identifying the three vertices bounding $f$ with the three vertices bounding the outer face of $T$ in such a way that vertex $c$ (which appears at the top in Figure 4) is mapped to the vertex of degree three (in B) of $f$. In the next paragraph, we will see why we do not have to specify how $a$ and $b$ are matched to $f$. The family $\mathcal{G}$ consists of all graphs on $5+6 \cdot 5=35$ vertices that can be obtained in this way. By construction all these graphs are planar 3-trees. Therefore by Lemma 10 on any given set of 35 points, the plane straight-line embedding is unique (if it exists), once the mapping for the outer face is determined.

Observe that $\mathcal{T}$ is flip-symmetric with respect to horizontal reflection. In other (more combinatorial) words, for every $T \in \mathcal{T}$ we can exchange the role of the bottom two vertices $a$ and $b$ of the outer face (and thereby also its orientation) to obtain a graph that is also in $\mathcal{T}$. The graphs form symmetric pairs of siblings $\left(T_{1}, T_{2}\right),\left(T_{3}, T_{4}\right),\left(T_{5}, T_{6}\right)$, and $T_{7}$ flips to itself. Therefore, regardless of the orientation in which we plant a graph from $\mathcal{T}$ onto a face of $\mathrm{B}$, we obtain a graph in $\mathcal{G}$, and so $\mathcal{G}$ is well-defined.

Next, we give a lower bound on the number of nonisomorphic graphs in $\mathcal{G}$.

\section{Lemma 13 The family $\mathcal{G}$ contains at least 9'805 pairwise nonisomorphic graphs.}

Proof. Consider the bipyramid B as a face-labeled object. There are $7^{6}$ different ways to assign a graph from $\mathcal{T}$ to each of the six now distinguishable faces. Denote this class of face-labeled graphs by $\mathcal{F}$. For many of these assignments the corresponding graphs are isomorphic if considered as abstract (unlabeled) graphs. However, the following argument shows that every isomorphism between two such graphs maps the vertex set of B to itself.

The two tips of $B$ have degree three and are incident to three faces. Onto each of the faces one graph from $\mathcal{T}$ is planted, which increases the degree by four (for $T_{1}, \ldots, T_{6}$ ) or three (for $T_{7}$ ) to a total of at least twelve. The three triangle vertices start with degree four and are incident to four faces. Every graph from $\mathcal{T}$ planted there adds at least one more edge, to a total degree of at least eight. But the highest degree among the interior vertices of the graphs in $\mathcal{T}$ is seven, which proves the claim.

Hence we have to look for isomorphisms only among the symmetries of the bipyramid B. The tips are distinguishable from the triangle vertices, because the former are incident to three high degree vertices, whereas the latter are incident to four high degree vertices. Selecting the mapping for one face of B determines the whole isomorphism. Since there are at most two ways to map a face to a face (we can select the mapping for the two non-tip vertices, that is, the orientation of the triangle), every graph in $\mathcal{F}$ is isomorphic to at most $2 \cdot 6=12$ graphs from $\mathcal{F}$. It follows that there are at least $7^{6} / 12>9^{\prime} 804$ pairwise nonisomorphic graphs in $\mathcal{G}$.

We now give an upper bound on the number of graphs of $\mathcal{G}$ that can be simultaneously embedded on a common point set.

Lemma 14 At most 7'392 pairwise nonisomorphic graphs of $\mathcal{G}$ admit a simultaneous (plane straight-line) embedding without mapping.

Proof. Consider a subset $\mathcal{G}^{\prime} \subseteq \mathcal{G}$ of pairwise nonisomorphic graphs and a point set $\mathrm{P}$ that admits a simultaneous embedding of $\mathcal{G}^{\prime}$. Since $\mathcal{G}^{\prime}$ is a class of maximal planar graphs, the convex hull of 
$P$ must be a triangle. For each $G \in \mathcal{G}^{\prime}$ we can select an outer face $f(G)$ and a mapping $\pi(G)$ for the vertices bounding $f(G)$ to the convex hull of $P$ so that the resulting straight-line embedding, which by Lemma 10 is completely determined by $f(G)$ and $\pi(G)$, is plane.

Let us group the graphs from $\mathcal{G}^{\prime}$ into bins, according to the maps $f$ and $\pi$. For $f$, there are $7 \cdot 11$ possible choices: one of the eleven faces of one of the seven graphs in $\mathcal{T}$. For $\pi$ there are three choices: one of the three possible rotations to map the face chosen by $f$ to the convex hull of $P$. Note that regarding $\pi$ there is no additional factor of two for the orientation of the face, because by flip-symmetry such a change corresponds to a different graph (for $T_{1}, \ldots, T_{6}$ ) or a different face of the graph (for $T_{7}$ ), that is, a different choice for $\mathrm{f}$. Altogether this yields a partition of $\mathcal{G}^{\prime}$ into $3 \cdot 77=231$ bins.

The crucial observation (and ultimate reason for this subdivision) is that for all graphs in a single bin the vertices of B (the bipyramid) are mapped to the same points. This is a consequence of the uniqueness of the embedding up to the mapping for the outer face (Lemma 10), which is identical for all graphs in the same bin. Therefore, the triangle $t$ of $B$ in which the outer face is located is mapped to the same oriented triple of points in $P$ for all graphs in the same bin. From there the pattern repeats, noting that every face of $B$ contains the same number of points (five) and that the polyhedron B is face-transitive so that there is no difference as to which face of B was selected to contain the outer face.

It follows that for all graphs in the same bin the graphs from $\mathcal{T}$ planted onto the faces of $B$ are mapped to the same point sets. Any two (nonisomorphic) graphs from $\mathcal{G}^{\prime}$ differ in at least one of those faces - and by definition not in the one in which the outer face was selected by $f$. In order for the graphs in a bin to be simultaneously embeddable on P, by Lemma 12 there are at most two different graphs from $\mathcal{T}$ mapped to any of the remaining five faces of $B$. Therefore there cannot be more than $2^{5}=32$ graphs from $\mathcal{G}^{\prime}$ in any bin. Hence $\left|\mathcal{G}^{\prime}\right| \leq 231 \cdot 32=7^{\prime} 392$, as claimed.

Since there are strictly more nonisomorphic graphs in $\mathcal{G}$ than can possibly be simultaneously embedded, not all graphs of $\mathcal{G}$ admit a simultaneous embedding. In particular, any subset of $7^{\prime} 392+1$ nonisomorphic graphs in $\mathcal{G}$ is a collection that does not have a simultaneous embedding. This proves our Theorem 2 .

\section{Small $n$-universal point sets}

As we have seen in the previous sections, there are no $n$-universal point sets of size $n$ for $n \geq 15$. In this section, we consider the case $n<15$. Specifically, we used a computer program [7] to show the following:

Theorem 15 There exist $n$-universal point sets of size $n$ for all $1 \leq n \leq 10$.

We use a straightforward brute-force approach. The two main ingredients are the aforementioned order type database [2] with point sets of size $n \leq 10$ and the plantri program for generating maximal planar graphs [5, 6]. To determine if a point set $P$ of size $n$ is $n$-universal, our program tests if for all maximal planar graphs $G=(V, E)$ on $n$ vertices, there exists a bijection $\varphi: V \rightarrow P$ such that straight-line drawing of $\mathrm{G}$ induced by $\varphi$ is plane. If such a bijection exists for all $G$, then $P$ is universal. Otherwise, there is a graph $G$ that has no plane straight-line embedding on $P$. Note that it is sufficient to consider maximal planar graphs since adding edges only makes the embedding problem more difficult. Work on the case $n=11$ is still in progress at the time of 
Table 1: The number of (non-equivalent) n-universal point sets of size $n$.

writing. For $n>11$ the approach unfortunately becomes infeasible; it is unknown whether or not there exist $n$-universal point sets of size $n$ for $11 \leq n \leq 14$. Table 1 gives an overview of the results of this paper and Figure 5 shows one universal point set for each $n=5, \ldots, 10$.
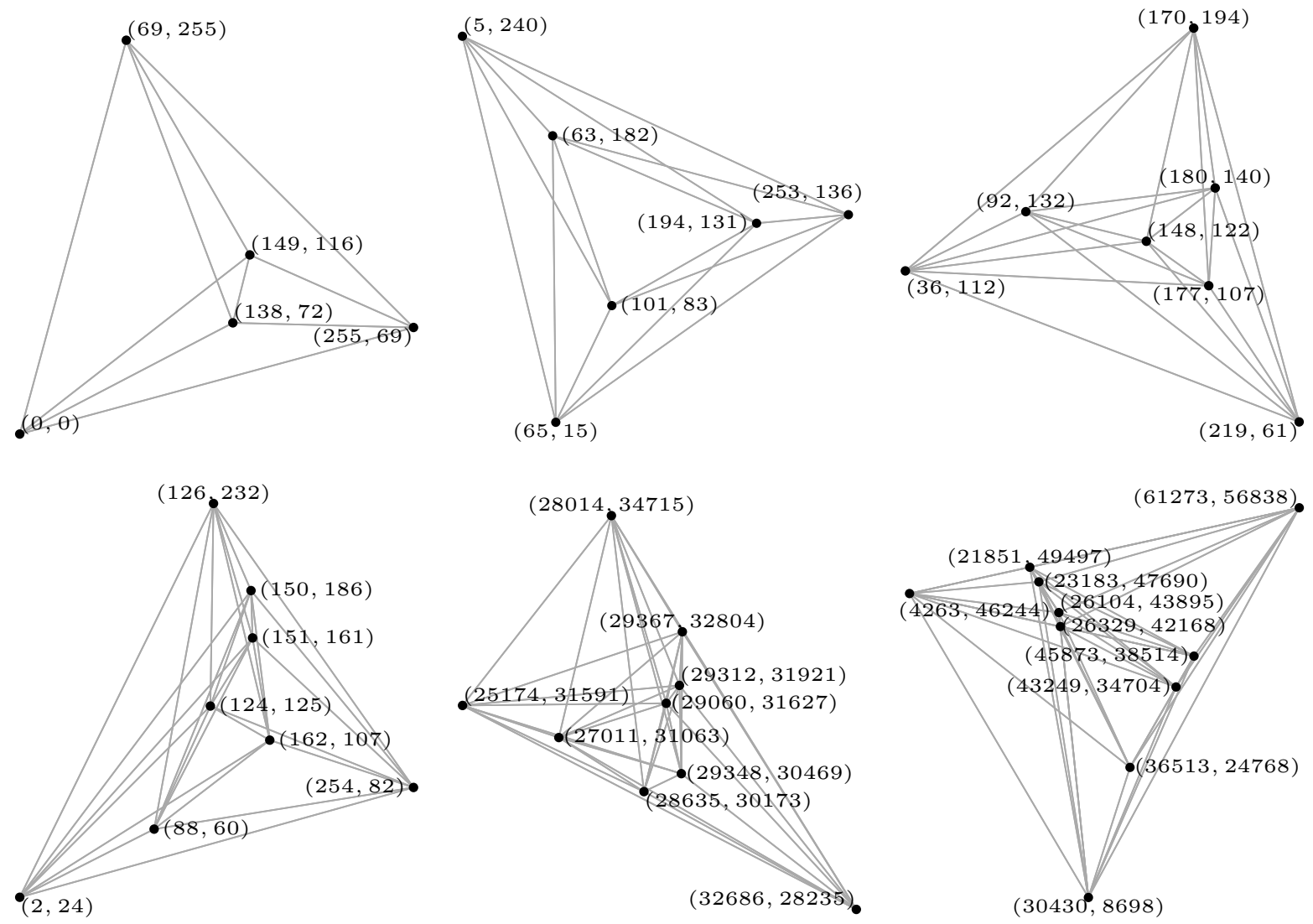

Figure 5: One universal point set for each $n=5, \ldots, 10$. Each pair of points is connected with a line segment. 


\section{References}

[1] Bernardo M. Ábrego and Silvia Fernández-Merchant. A lower bound for the rectilinear crossing number. Graphs and Combinatorics, 21(3):293-300, 2005.

[2] Oswin Aichholzer and Hannes Krasser. The point set order type data base: A collection of applications and results. In Proc. 13th Canad. Conf. Comput. Geom., pages 17-20, Waterloo, Canada, 2001.

[3] Lowell W. Beineke and Raymond E. Pippert. Enumerating dissectible polyhedra by their automorphism groups. Canad. J. Math., 26:50-67, 1974.

[4] Peter Brass, Eowyn Cenek, Cristian A. Duncan, Alon Efrat, Cesim Erten, Dan P. Ismailescu, Stephen G. Kobourov, Anna Lubiw, and Joseph S.B. Mitchell. On simultaneous planar graph embeddings. Comput. Geom. Theory Appl., 36(2):117-130, 2007.

[5] Gunnar Brinkmann and Brendan McKay. Fast generation of planar graphs. MATCH Communications in Mathematical and in Computer Chemistry, 58(2):323-357, 2007.

[6] Gunnar Brinkmann and Brendan McKay. The program plantri. http://cs.anu.edu.au/ ־bdm/plantri, 2007.

[7] Jean Cardinal, Michael Hoffmann, and Vincent Kusters. A program to find all universal point sets. http://people.inf.ethz.ch/kustersv/universal.html, 2013.

[8] Marek Chrobak and Howard J. Karloff. A lower bound on the size of universal sets for planar graphs. SIGACT News, 20(4):83-86, 1989.

[9] Hubert de Fraysseix, János Pach, and Richard Pollack. How to draw a planar graph on a grid. Combinatorica, 10(1):41-51, 1990.

[10] Erik D. Demaine, Joseph S. B. Mitchell, and Joseph O'Rourke. The Open Problems Project, Problem \#45. http://maven.smith.edu/ orourke/TOPP/P45.html.

[11] István Fáry. On straight lines representation of planar graphs. Acta Sci. Math. Szeged, 11:229-233, 1948.

[12] Radoslav Fulek and Csaba D. Tóth. Universal point sets for planar three-trees. CoRR, abs/1212.6148, 2012.

[13] Jacob E. Goodman and Richard Pollack. Multidimensional sorting. SIAM J. Comput., 12(3):484-507, 1983.

[14] Stephen G. Kobourov. personal communication, 2012.

[15] Maciej Kurowski. A 1.235 lower bound on the number of points needed to draw all n-vertex planar graphs. Information Processing Letters, 92(2):95-98, 2004.

[16] László Lovász, Katalin Vesztergombi, Uli Wagner, and Emo Welzl. Convex quadrilaterals and k-sets. In János Pach, editor, Towards a Theory of Geometric Graphs, volume 324 of Contemporary Mathematics, pages 139-148. American Mathematical Society, Providence, RI, 2004. 
[17] Walter Schnyder. Embedding planar graphs on the grid. In Proc. 1st ACM-SIAM Sympos. Discrete Algorithms, pages 138-148, 1990.

[18] Klaus Wagner. Bemerkungen zum Vierfarbenproblem. Jahresbericht der Deutschen Mathematiker-Vereinigung, 46:26-32, 1936. 